\title{
Pacific
}

Journal of

Mathematics

\section{TENSOR PRODUCTS WITH ANISOTROPIC PRINCIPAL SERIES REPRESENTATIONS OF FREE GROUPS}

\section{Carlo Pensavalle and Tim Steger}




\title{
TENSOR PRODUCTS WITH ANISOTROPIC PRINCIPAL SERIES REPRESENTATIONS OF FREE GROUPS
}

\author{
Carlo Pensavalle and Tim Steger
}

\begin{abstract}
Let $\tau$ be the tensor product of an anisotropic principal series representation of a free group $\Gamma$, not an endpoint representation, with an irreducible unitary finite dimensional $\Gamma$-representation. Usually $\tau$ is irreducible and has exactly two perfect boundary realizations. In a certain well specified anomalous case $\tau$ splits into two irreducible components and each component has exactly one boundary realization, which is not perfect.
\end{abstract}

\section{Introduction.}

Let $\Gamma$ be a noncommutative free group on finitely many generators. Fix a basis $A_{+}$for $\Gamma$ and let $A=A_{+} \cup A_{+}^{-1}$ consist of the basis elements and their inverses. Let $\pi$ be a representation from one of the anisotropic principal series of [Figà-Talamanca-Steger]. Let $\rho$ be an irreducible unitary finite dimensional representation of $\Gamma$. The aim of this paper is to apply the results of [Steger] to the tensor product $\pi \otimes \rho$. In particular, we show that the representation $\pi \otimes \rho$ is irreducible under most circumstances, and decomposes into two irreducible components when it does decompose.

Anisotropic principal series representations. The following is taken from [Figà-Talamanca-Steger]. (That paper deals with a free product of copies of $\mathbf{Z} / 2$. The case of a free group is somewhat less complicated.) Let $\mu$ be a symmetric probability measure on $A$, suppose that $\mu(a)>0$ for $a \in A$, and denote by $\operatorname{Spec}(\mu)$ the spectrum of $\mu$ acting on $\ell^{2}(\Gamma)$ by left convolution. Then $\operatorname{Spec}(\mu)$ consists of a closed interval symmetric about 0 . Let $\sigma$ belong to the interior of that interval. There is a unitary representation, $\pi$, described below, determined by the pair $(\mu, \sigma)$. Fixing $\mu$ and letting $\sigma$ vary we obtain a series of representations whose direct integral is the regular representation of $\Gamma$. Call such a series an anisotropic principal series for $(\Gamma, A)$, and call the representation $\pi$ an anisotropic principal series representation.

Fix $(\mu, \sigma)$. Recall that each $x \in \Gamma$ has a unique shortest expression, $a_{1} a_{2} \cdots a_{L}$, as a product of elements in $A$. That shortest expression is the reduced word for $x$, characterized by the property that $a_{l} a_{l+1} \neq e$ for any $l$. 
The length, $|x|$, of $x$ as above is $L$. A nonzero function $f: \Gamma \rightarrow \mathbf{C}$ is called multiplicative if

$$
f(x y)=f(x) f(y) \quad \text { when }|x y|=|x|+|y| .
$$

A multiplicative function is determined by its values on $A$. For $\gamma \notin \operatorname{Spec}(\mu)$, the inverse of left convolution by $\gamma \delta_{e}-\mu$ on $\ell^{2}(\Gamma)$ is left convolution by $g_{\gamma}=h_{\gamma} /\left(2 w_{\gamma}\right)$, where $h_{\gamma}$ is a multiplicative function and $w_{\gamma}$ is a constant. Let

$$
\begin{aligned}
h_{ \pm} & =h_{\sigma \pm i 0}=\lim _{\delta \rightarrow 0+} h_{\sigma \pm i \delta} \\
w_{ \pm} & =w_{\sigma \pm i 0}=\lim _{\delta \rightarrow 0+} w_{\sigma \pm i \delta} \\
g_{ \pm} & =g_{\sigma \pm i 0}=h_{ \pm} /\left(2 w_{ \pm}\right) \\
\phi & =\phi_{\sigma}=\left(g_{+}-g_{-}\right) /\left(g_{+}(e)-g_{-}(e)\right) .
\end{aligned}
$$

Then $\phi$ is a positive definite function on $\Gamma$, and the representation $(\pi, \mathcal{H})$ is defined by applying the construction of Gel'fand and Raikov to $\phi$.

For later use, observe that since $\mu$ is symmetric, $h_{\gamma}(a)=h_{\gamma}\left(a^{-1}\right)$ for all $a \in A$, and consequently the same holds for $h_{ \pm}$. Also note that since $\mu$ is real, $h_{\bar{\gamma}}=\bar{h}_{\gamma}, w_{\bar{\gamma}}=\bar{w}_{\gamma}$, and consequently $h_{ \pm}, w_{ \pm}$, and $g_{ \pm}$are complex conjugates of one another.

Results for $\tau=\pi \otimes \rho$. Let $\mathcal{H} \otimes \mathcal{V}$ be the representation space of $\tau$, where $\mathcal{H}$ is the representation space of $\pi$ and $\mathcal{V}$ is the representation space of $\rho$. Define sgn: $\Gamma \longrightarrow \mathbf{C}^{\times}$by $\operatorname{sgn}(x)=(-1)^{|x|}$.

Theorem 1.1. Exclude the anomalous case that $\sigma=0$ and $\rho \cong \rho \otimes \operatorname{sgn}$. Then $\tau$ is irreducible.

In the anomalous case, $\tau$ splits into two irreducible representations. The full story of the anomalous case is in Section 4.

Theorem 1.2. Exclude the anomalous case that $\sigma=0$ and $\rho \cong \rho \otimes \operatorname{sgn}$. Then there is a dense subspace $\mathcal{Z} \subseteq \mathcal{H} \otimes \mathcal{V}$ and a constant $C_{0}>0$ so that

$$
\lim _{\epsilon \rightarrow 0+} C_{0} \epsilon \sum_{x \in \Gamma} e^{-\epsilon|x|}\left\langle w_{1}, \tau(x) w_{2}\right\rangle \overline{\left\langle w_{3}, \tau(x) w_{4}\right\rangle}=\left\langle w_{1}, w_{3}\right\rangle \overline{\left\langle w_{2}, w_{4}\right\rangle}
$$

for $w_{1}, w_{3} \in \mathcal{H} \otimes \mathcal{V}$ and $w_{2}, w_{4} \in \mathcal{Z}$.

The boundary, $\Omega$, of $\Gamma$. The statements of the other main results, and the proofs of all the results, depend on the idea of the boundary of $\Gamma$. See [FigàTalamanca-Nebbia] or the introduction to [Kuhn-Steger] for more detailed discussions of the boundary. Give $\Gamma$ the structure of a tree by putting 
an edge between $x$ and $x a$ for $x \in \Gamma$ and $a \in A$. Let $d(\cdot, \cdot)$ be the distance on $\Gamma$ given by this tree structure. Note that $|x|=d(e, x)$. A geodesic is a sequence $\left(x_{l}\right)_{l} \subseteq \Gamma$ such that $d\left(x_{l}, x_{m}\right)=|l-m|$. There is a unique geodesic between any two elements of $\Gamma$. The boundary, $\Omega$, is conveniently defined as \{semiinfinite geodesics $\left.\left(x_{l}\right)_{l=0}^{\infty}\right\} / \sim$ where two such geodesics are considered equivalent when some shift of the indices makes them cofinally equal. This space is also called the space of ends of the tree. Given $x \in \Gamma$ and $\omega \in \Omega$, there is a unique geodesic $[x, \omega)$ starting at $x$ and representing $\omega$, and this we think of as the geodesic from $x$ to $\omega$. Let

$$
[e, \omega)=\left(e, \omega_{1}, \omega_{2}, \ldots\right) .
$$

There will exist $\left(a_{l}\right)_{l=1}^{\infty} \subseteq A$ satisfying $a_{l} a_{l+1} \neq e$ such that $\omega_{L}=a_{1} a_{2} \cdots a_{L}$. We may think of $\omega$ as the infinite reduced word $a_{1} a_{2} a_{3} \ldots$ For $z \in \Gamma$, define

$$
\Gamma(z)=\{x \in \Gamma ; z \in[e, x]\} \quad \text { and } \quad \Omega(z)=\{\omega \in \Omega ; z \in[e, \omega)\}
$$

those reduced words, respectively finite or infinite, which start out with the word for $z$. Topologize $\Gamma \cup \Omega$ by taking the singleton subsets of $\Gamma$ and the sets $(\Gamma(z) \cup \Omega(z))_{z \in \Gamma}$ as a basis of open sets. This topology makes $\Gamma \cup \Omega$ and $\Omega$ compact, with $\Gamma$ discrete and with every point of $\Omega$ in the closure of $\Gamma$. Indeed, $\omega=\lim _{l \rightarrow \infty} \omega_{l}$ where $\omega_{l}$ is as in (1.3).

The left action of $\Gamma$ on itself preserves the tree structure, and therefore it induces an action of $\Gamma$ on $\Omega$. We write that action as multiplication, and indeed, it is the obvious action by left multiplication of finite reduced words on infinite reduced words. This left action of $\Gamma$ on $\Gamma \cup \Omega$ leaves the topology fixed.

Realization of $\pi$ on the boundary. Define a probability measure $\nu$ on $\Omega$ by

$$
\nu(\Omega(x a))=\left|h_{+}(x a)\right|^{2} /\left(1+\left|h_{+}(a)\right|^{2}\right) \quad \text { for } x \in \Gamma, a \in A,|x a|=|x|+1 .
$$

This works since $\sum_{a \in A}\left|h_{+}(a)\right|^{2} /\left(1+\left|h_{+}(a)\right|^{2}\right)=1$. Define a unitary representation $\pi_{+\Gamma}^{\prime}$ of $\Gamma$ on $L^{2}(\Omega, d \nu)$ by

$$
\left(\pi_{+\Gamma}^{\prime}(a) F\right)(\omega)= \begin{cases}h_{+}(a) F\left(a^{-1} \omega\right), & \text { if } \omega \notin \Omega(a) \\ h_{+}(a)^{-1} F\left(a^{-1} \omega\right), & \text { if } \omega \in \Omega(a)\end{cases}
$$

for $a \in A$. This extends to a representation of $\Gamma$ because $\pi_{+\Gamma}^{\prime}(a) \pi_{+\Gamma}^{\prime}\left(a^{-1}\right)=1$. The action of general $x \in \Gamma$ is given by

$$
\left(\pi_{+\Gamma}^{\prime}(x) F\right)(\omega)=P_{+}(x, \omega) F\left(x^{-1} \omega\right)
$$


for

$$
P_{+}(x, \omega)=\frac{h_{+}\left(x_{2}\right)}{h_{+}\left(x_{1}\right)}
$$

where $x=x_{1} x_{2}$ and $x_{1}$ is the last element common to the geodesics $[e, x]$ and $[e, \omega)$, that is, the longest common initial segment of the reduced words for $x$ and $\omega$. Unitarity of $\pi_{+\Gamma}^{\prime}$ depends on

$$
\left|P_{+}(x, \omega)\right|^{2}=\frac{d \nu\left(x^{-1} \omega\right)}{d \nu(\omega)}
$$

the right hand side being a Radon-Nikodym derivative. This is easy to check for $x \in A$ and then follows for all $x$. One defines $\pi_{-\Gamma}^{\prime}$ in just the same way, using $h_{-}$instead of $h_{+}$.

[Figà-Talamanca-Steger] exhibits unitary $\Gamma$-maps $\iota_{ \pm}: \mathcal{H} \longrightarrow L^{2}(\Omega, d \nu)$, intertwining $\pi$ to $\pi_{ \pm \Gamma}^{\prime}$ respectively. Also exhibited explicitly is the intertwining operator

$$
J=\iota_{-} \iota_{+}^{*}: L^{2}(\Omega, d \nu) \longrightarrow L^{2}(\Omega, d \nu)
$$

which satisfies $J \pi_{+\Gamma}^{\prime}(x)=\pi_{-\Gamma}^{\prime}(x) J$ for $x \in \Gamma$. Although complex conjugation intertwines $\pi_{+\Gamma}^{\prime}$ and $\pi_{-\Gamma}^{\prime}$ just as $J$ does, $J$ is a complex linear map, more complicated than simple conjugation.

Boundary representations. Define $*$-representations $\pi_{ \pm \Omega}^{\prime}$ of the commutative $C^{*}$-algebra $C(\Omega)$ on $L^{2}(\Omega, d \nu)$ by

$$
\left(\pi_{+\Omega}^{\prime}(G) F\right)(\omega)=\left(\pi_{-\Omega}^{\prime}(G) F\right)(\omega)=G(\omega) F(\omega) .
$$

The symbols $\pi_{ \pm \Gamma}^{\prime}$ and $\pi_{ \pm \Omega}^{\prime}$ are chosen so as to match the following definition from [Steger].

Definition 1.3. Let $\Gamma$ be any free group and let $\Omega$ be its boundary. A $(\Gamma, C(\Omega))$-representation or boundary representation, $\pi^{\prime}$, of $\Gamma$ on $\mathcal{H}^{\prime}$ is a pair $\left(\pi_{\Gamma}^{\prime}, \pi_{\Omega}^{\prime}\right)$ such that

(1) $\pi_{\Gamma}^{\prime}$ is a unitary representation of $\Gamma$ on $\mathcal{H}^{\prime}$.

(2) $\pi_{\Omega}^{\prime}$ is a $*$-representation of $C(\Omega)$ on $\mathcal{H}^{\prime}$.

(3) For $x \in \Gamma$ and $G \in C(\Omega), \pi_{\Gamma}^{\prime}(x) \pi_{\Omega}^{\prime}(G) \pi_{\Gamma}^{\prime}\left(x^{-1}\right)=\pi_{\Omega}^{\prime}(\lambda(x) G)$.

Here $(\lambda(x) G)(\omega)=G\left(x^{-1} \omega\right)$. The reader familiar with crossed product $C^{*}$-algebras will see that a boundary representation is just a representation of the crossed product algebra $\Gamma \ltimes C(\Omega)$. We will routinely drop the subscripts $\Gamma$ and $\Omega$ on $\pi_{ \pm}^{\prime}$. One may easily verify that the two representations $\pi_{+}^{\prime}$ defined by (1.4) and (1.7) fit together to give a boundary representation of $\Gamma$. One makes $\pi_{-}^{\prime}$ into a boundary representation in the same way. Note 
that although $\pi_{+}^{\prime}$ and $\pi_{-}^{\prime}$ are equivalent as $\Gamma$-representations and identical as $C(\Omega)$-representations, they are not equivalent as $(\Gamma, C(\Omega))$-representations.

Let $\rho$ be an irreducible unitary representation of $\Gamma$ on a finite dimensional Hilbert space $\mathcal{V}$. It is easily verified that the definitions

$$
\tau_{ \pm \Gamma}^{\prime}(x)=\pi_{ \pm \Gamma}^{\prime}(x) \otimes \rho(x) \quad \tau_{ \pm \Omega}^{\prime}(G)=\pi_{ \pm \Omega}^{\prime}(G) \otimes \mathrm{id}
$$

give boundary representations $\tau_{ \pm}^{\prime}$ acting on $L^{2}(\Omega, d \nu) \otimes \mathcal{V}$. Indeed this simple construction applies generally, yielding a boundary representation as the tensor product of any boundary representation and any ordinary $\Gamma$ representation. Identifying $L^{2}(\Omega, d \nu) \otimes \mathcal{V}$ with $L^{2}(\Omega, d \nu ; \mathcal{V})$, (see [ReedSimon] Section 2.4, Theorem 10,) gives for any $F \in L^{2}(\Omega, d \nu ; \mathcal{V})$

$$
\left(\tau_{ \pm \Gamma}^{\prime}(x) F\right)(\omega)=P_{ \pm}(x, \omega) \rho(x) F\left(x^{-1} \omega\right)
$$

and

$$
\left(\tau_{ \pm \Omega}^{\prime}(G) F\right)(\omega)=G(\omega) F(\omega)
$$

where $x \in \Gamma$ and $G \in C(\Omega)$.

The following definitions are also from [Steger].

Definition 1.4. Let $\Gamma$ be a free group, let $\Omega$ be its boundary, and let $\pi$ be a fixed unitary representation of $\Gamma$ on $\mathcal{H}$. A boundary intertwiner for $\pi$ is a pair $\left(\iota, \pi^{\prime}\right)$ satisfying

(1) $\pi^{\prime}$ is a boundary representation of $\Gamma$ on a Hilbert space $\mathcal{H}^{\prime}$.

(2) $\iota$ is a bounded $\Gamma$-map from $\mathcal{H}$ to $\mathcal{H}^{\prime}$.

(3) $\mathcal{H}^{\prime}$ is generated as a $(\Gamma, C(\Omega))$-space by $\iota(\mathcal{H})$.

If $\iota$ is an isometric inclusion, the pair is called a boundary realization of $\pi$, and if $\iota$ is unitary, the pair is called a perfect boundary realization of $\pi$. Condition (3) is equivalent to requiring that $\pi^{\prime}(C(\Omega)) \iota(\mathcal{H})$ be dense in $\mathcal{H}^{\prime}$.

Definition 1.5. Two boundary intertwiners $\left(\iota_{1}, \pi_{1}^{\prime}\right)$ and $\left(\iota_{2}, \pi_{2}^{\prime}\right)$ for the same given representation $\pi$ of $\Gamma$ are equivalent if there is a unitary $(\Gamma, C(\Omega))$-map $U: \mathcal{H}_{1}^{\prime} \longrightarrow \mathcal{H}_{2}^{\prime}$ so that $U \iota_{1}=\iota_{2}$.

Let $\left(\iota_{1}, \pi_{1}^{\prime}\right)$ and $\left(\iota_{2}, \pi_{2}^{\prime}\right)$ be two boundary intertwiners for a given representation $\pi$ of $\Gamma$. The direct sum of these two intertwiners,

$$
\left(\iota_{s}, \pi_{s}^{\prime}\right)=\left(\iota_{1}, \pi_{1}^{\prime}\right)+\left(\iota_{2}, \pi_{2}^{\prime}\right)
$$

is defined in two steps. Let $\tilde{\pi}_{s}^{\prime}$ be the direct sum boundary representation on $\mathcal{H}_{1}^{\prime} \oplus \mathcal{H}_{2}^{\prime}$. Let $\tilde{\iota}_{s}(v)=\left(\iota_{1}(v), \iota_{2}(v)\right)$. Then $\left(\tilde{\iota}_{s}, \tilde{\pi}_{s}^{\prime}\right)$ satisfies the definition of a boundary intertwiner except for condition (3). Let $\mathcal{H}_{s}^{\prime}$ be the closure in $\mathcal{H}_{1}^{\prime} \oplus \mathcal{H}_{2}^{\prime}$ of $\tilde{\pi}_{s}^{\prime}(C(\Omega)) \tilde{\iota}_{s}(\mathcal{H})$. Then $\mathcal{H}_{s}^{\prime}$ is a subboundary representation of $\mathcal{H}_{1}^{\prime} \oplus \mathcal{H}_{2}^{\prime}$. Denote the boundary representation on $\mathcal{H}_{s}^{\prime}$ by $\pi_{s}^{\prime}$. Finally, let $\iota_{s}: \mathcal{H} \longrightarrow \mathcal{H}_{s}^{\prime}$ be $\tilde{\iota}_{s}$ with its range restricted to $\mathcal{H}_{s}^{\prime}$. 
For $G \in C(\Omega)$ one may verify that

$$
\iota_{s}^{*} \pi_{s}^{\prime}(G) \iota_{s}=\iota_{1}^{*} \pi_{1}^{\prime}(G) \iota_{1}+\iota_{2}^{*} \pi_{2}^{\prime}(G) \iota_{2} .
$$

To be consistent with that definition define, for a boundary intertwiner $\left(\iota, \pi^{\prime}\right)$ and for real $t>0$,

$$
t\left(\iota, \pi^{\prime}\right)=\left(t^{1 / 2} \iota, \pi^{\prime}\right)
$$

and define

$$
0\left(\iota, \pi^{\prime}\right)=\left(0, \zeta^{\prime}\right)
$$

where $\zeta^{\prime}$ is the boundary representation on 0 -dimensional Hilbert space. From these definitions it follows that if $\left(\iota_{1}, \pi_{1}^{\prime}\right)$ and $\left(\iota_{2}, \pi_{2}^{\prime}\right)$ are boundary realizations, that is, if $\iota_{1}$ and $\iota_{2}$ are isometric inclusions, and if $0 \leq t \leq 1$, then $t\left(\iota_{1}, \pi_{1}^{\prime}\right)+(1-t)\left(\iota_{2}, \pi_{2}^{\prime}\right)$ is also a boundary realization.

Results on boundary realizations of $\tau$. Return to the specific case of the tensor product representation $(\tau, \mathcal{H} \otimes \mathcal{V})$ of an anisotropic principal series representation $(\pi, \mathcal{H})$ and an irreducible unitary finite dimensional representation $(\rho, \mathcal{V})$ of $\Gamma$. The maps

$$
\iota_{ \pm} \otimes \mathrm{id}: \mathcal{H} \otimes \mathcal{V} \longrightarrow L^{2}(\Omega, d \nu) \otimes \mathcal{V}
$$

are unitary $\Gamma$-maps intertwining $\tau$ with $\tau_{ \pm \Gamma}^{\prime}$. Thus $\left(\iota_{ \pm} \otimes \mathrm{id}, \tau_{ \pm}^{\prime}\right)$, where $\tau_{ \pm}^{\prime}=$ $\left(\tau_{ \pm \Gamma}^{\prime}, \tau_{ \pm \Omega}^{\prime}\right)$, are two perfect boundary realizations of $\tau$.

Theorem 1.6. Exclude the anomalous case that $\sigma=0$ and $\rho \cong \rho \otimes \operatorname{sgn}$. Then, up to equivalence, the only perfect boundary realizations of $\tau$ are $\left(\iota_{ \pm} \otimes\right.$ $\left.\mathrm{id}, \tau_{ \pm}^{\prime}\right)$. Moreover, all boundary realizations, perfect or otherwise, are given, up to equivalence, by

$$
t\left(\iota_{+} \otimes \mathrm{id}, \tau_{+}^{\prime}\right)+(1-t)\left(\iota_{-} \otimes \mathrm{id}, \tau_{-}^{\prime}\right) \quad \text { for } 0 \leq t \leq 1 .
$$

Outline. Section 2 describes some general results on unitary representations of free groups and their boundary realizations. Section 3 establishes the applicability of those general results in the present context. Section 4 deals with the anomalous case, $\sigma=0$ and $\rho \cong \rho \otimes \operatorname{sgn}$.

\section{General Results.}

If $\mathcal{H}$ and $\mathcal{H}_{1}$ are Hilbert spaces, we denote by $\mathcal{B}\left(\mathcal{H}, \mathcal{H}_{1}\right)$ the vector space of all bounded linear maps from $\mathcal{H}$ to $\mathcal{H}_{1}$. If $\mathcal{H}=\mathcal{H}_{1}$ then $\mathcal{B}(\mathcal{H}) \equiv \mathcal{B}(\mathcal{H}, \mathcal{H})$.

Definition 2.1. Let $T \in \mathcal{B}(\mathcal{H})$. We say that $T$ is positive if

$$
\langle T v, v\rangle_{\mathcal{H}} \geq 0 \quad \text { for } v \in \mathcal{H} .
$$


We denote by $\mathcal{B}^{+}(\mathcal{H})$ the set of all positive operators in $\mathcal{B}(\mathcal{H})$.

Definition 2.2. Let $T \in \mathcal{B}^{+}(\mathcal{H})$. We define the trace of $T$ as

$$
\operatorname{tr}(T)=\sum_{n=1}^{+\infty}\left\langle T e_{n}, e_{n}\right\rangle_{\mathcal{H}}
$$

where $\left(e_{n}\right)_{n=1}^{+\infty}$ is a fixed orthonormal basis for $\mathcal{H}$.

Properties 2.3. Let $S$ and $T$ be in $\mathcal{B}^{+}(\mathcal{H})$.

(1) $\operatorname{tr}(T) \in[0,+\infty]$.

(2) $\operatorname{tr}(\alpha S+\beta T)=\alpha \operatorname{tr}(S)+\beta \operatorname{tr}(T)$ for $\alpha, \beta \in \mathbf{R}^{+}$.

(3) $\operatorname{tr}\left(S S^{*}\right)=\|S\|_{H S}^{2}$ where $\|\cdot\|_{H S}$ is the Hilbert-Schmidt norm.

(4) If $U \in \mathcal{B}(\mathcal{H})$ is unitary, then $\operatorname{tr}\left(U T U^{-1}\right)=\operatorname{tr}(T)$.

(5) $\operatorname{tr}(T)$ is independent of the choice of basis.

(6) Let $\mathcal{V}$ be a finite dimensional Hilbert space, then $\operatorname{tr}\left(T \otimes \operatorname{id}_{\mathcal{V}}\right)=\operatorname{dim} \mathcal{V} \operatorname{tr}(T)$

Proof. For (1)-(5), see [Dixmier] Section 1.6.6, Theorem 5. Let $\left(v_{m}\right)_{m=1}^{N}$ be an orthonormal basis of $\mathcal{V}$. Then using $\left(e_{n} \otimes v_{m}\right)_{n, m}$ as a basis of $\mathcal{H} \otimes \mathcal{V}$ gives (6).

Definition 2.4. Let $S, T \in \mathcal{B}^{+}(\mathcal{H})$. We define the following inner product

$$
(S, T)_{B^{+}(\mathcal{H})}=\operatorname{tr}(\sqrt{S} T \sqrt{S}) .
$$

Properties 2.5. Let $S$ and $T$ be in $\mathcal{B}^{+}(\mathcal{H})$.

(1) $(S, T)_{\mathcal{B}^{+}(\mathcal{H})} \in[0,+\infty]$.

(2) $(S, T)_{\mathcal{B}^{+}(\mathcal{H})}=\|\sqrt{S} \sqrt{T}\|_{H S}^{2}$.

(3) $(S, T)_{\mathcal{B}^{+}(\mathcal{H})}=(T, S)_{\mathcal{B}^{+}{ }_{(\mathcal{H})}}$.

(4) $(S, T)_{\mathcal{B}^{+}(\mathcal{H})}$ is bilinear in $S$ and $T$.

(5) Let $\mathcal{V}$ be a finite dimensional Hilbert space, then $(S \otimes \mathrm{id}, T \otimes \mathrm{id})_{\mathcal{B}^{+}(\mathcal{H} \otimes \mathcal{V})}=$ $\operatorname{dim} \mathcal{V}(S, T)_{\mathcal{B}^{+}(\mathcal{H})}$.

Proof. (1) follows directly from the definition. For (2),

$$
\begin{aligned}
(S, T)_{\mathcal{B}^{+}(\mathcal{H})} & =\operatorname{tr}(\sqrt{S} T \sqrt{S})=\operatorname{tr}(\sqrt{S} \sqrt{T} \sqrt{T} \sqrt{S}) \\
& =\operatorname{tr}\left((\sqrt{S} \sqrt{T})(\sqrt{S} \sqrt{T})^{*}\right)=\|\sqrt{S} \sqrt{T}\|_{H S}^{2} .
\end{aligned}
$$


For (3),

$$
(S, T)_{\mathcal{B}^{+}(\mathcal{H})}=\|\sqrt{S} \sqrt{T}\|_{H S}^{2}=\left\|(\sqrt{S} \sqrt{T})^{*}\right\|_{H S}^{2}=\|\sqrt{T} \sqrt{S}\|_{H S}^{2}=(T, S)_{\mathcal{B}^{+}(\mathcal{H})} .
$$

Finally (4) follows from the definition and (3), and (5) follows from (6) of Properties 2.3.

One may easily verify the following lemma.

Lemma 2.6. Let $\Gamma$ be a free group. Let $\pi$ be a unitary representation of $\Gamma$ and let $\left(\iota, \pi^{\prime}\right)$ be a boundary intertwiner for $\pi$. For any $G \in C(\Omega)$ with $G \geq 0$ the operator

$$
\iota^{*} \pi^{\prime}(G) \iota: \mathcal{H} \longrightarrow \mathcal{H}
$$

is linear, bounded and positive.

Now we can give the definition of the Finite Trace Condition (FTC) for boundary intertwiners.

Definition 2.7. Let $A$ be a set of generators and their inverses for a free group $\Gamma$. Let $\left(\iota_{1}, \pi_{1}^{\prime}\right)$ and $\left(\iota_{2}, \pi_{2}^{\prime}\right)$ be two boundary intertwiners for a representation $(\pi, \mathcal{H})$ of $\Gamma$. We say that $\left(\iota_{1}, \pi_{1}^{\prime}\right)$ and $\left(\iota_{2}, \pi_{2}^{\prime}\right)$ satisfy the (FTC) if

$$
\left(\iota_{1}^{*} \pi_{1}^{\prime}\left(\mathbf{1}_{\Omega(a)}\right) \iota_{1}, \iota_{2}^{*} \pi_{2}^{\prime}\left(\mathbf{1}-\mathbf{1}_{\Omega(a)}\right) \iota_{2}\right)_{\mathcal{B}^{+}(\mathcal{H})}<+\infty
$$

for all $a \in A$.

Lemma 2.8. Let $\Gamma$ be a free group with $A$ as a set of generators and inverses. Let $\pi$ be a unitary representation of $\Gamma$ and let $\left(\iota_{1}, \pi_{1}^{\prime}\right)$ and $\left(\iota_{2}, \pi_{2}^{\prime}\right)$ be boundary intertwiners for $\Gamma$. Let $\rho$ be a unitary representation of $\Gamma$ on a finite dimensional Hilbert space $\mathcal{V}$. Let $\tau=\pi \otimes \rho, \tau_{1, \Gamma}^{\prime}=\pi_{1, \Gamma}^{\prime} \otimes \rho, \tau_{2, \Gamma}^{\prime}=\pi_{2, \Gamma}^{\prime} \otimes \rho$, $\tau_{1, \Omega}^{\prime}=\pi_{1, \Omega}^{\prime} \otimes \mathrm{id}$, and $\tau_{2, \Omega}^{\prime}=\pi_{2, \Omega}^{\prime} \otimes \mathrm{id}$. Then $\left(\iota_{1} \otimes \mathrm{id}, \tau_{1}^{\prime}\right)$ and $\left(\iota_{2} \otimes \mathrm{id}, \tau_{2}^{\prime}\right)$ satisfy the $(F T C)$ as realizations of $\tau$ if and only if $\left(\iota_{1}, \pi_{1}^{\prime}\right)$ and $\left(\iota_{2}, \pi_{2}^{\prime}\right)$ satisfy the (FTC) as realizations of $\pi$.

Proof. Let $a \in A$. Then

$$
\begin{aligned}
& \left(\left(\iota_{1} \otimes \mathrm{id}\right)^{*} \tau_{1}^{\prime}\left(\mathbf{1}_{\Omega(a)}\right)\left(\iota_{1} \otimes \mathrm{id}\right),\left(\iota_{2} \otimes \mathrm{id}\right)^{*} \tau_{2}^{\prime}\left(\mathbf{1}-\mathbf{1}_{\Omega(a)}\right)\left(\iota_{2} \otimes \mathrm{id}\right)\right)_{\mathcal{B}^{+}(\mathcal{H} \otimes \mathcal{V})} \\
& =\left(\iota_{1}^{*} \pi_{1}^{\prime}\left(\mathbf{1}_{\Omega(a)}\right) \iota_{1} \otimes \mathrm{id}, \iota_{2}^{*} \pi_{2}^{\prime}\left(\mathbf{1}-\mathbf{1}_{\Omega(a)}\right) \iota_{2} \otimes \mathrm{id}\right)_{\mathcal{B}^{+}(\mathcal{H} \otimes \mathcal{V})} \\
& =\operatorname{dim} \mathcal{V}\left(\iota_{1}^{*} \pi_{1}^{\prime}\left(\mathbf{1}_{\Omega(a)}\right) \iota_{1}, \iota_{2}^{*} \pi_{2}^{\prime}\left(\mathbf{1}-\mathbf{1}_{\Omega(a)}\right) \iota_{2}\right)_{\mathcal{B}^{+}(\mathcal{H})}
\end{aligned}
$$

according to (5) of Properties 2.5.

The following result, which is our main tool, is from [Steger]. 
Theorem 2.9. Let $\Gamma$ be a free group and let $A$ be a set of generators and their inverses. Let $(\pi, \mathcal{H})$ be a unitary representation of $\Gamma$ on a Hilbert space $\mathcal{H}$. If $\left(\iota_{1}, \pi_{1}^{\prime}\right)$ and $\left(\iota_{2}, \pi_{2}^{\prime}\right)$ are two perfect boundary realizations of $\pi$ satisfying the $(F T C)$, and if $\pi_{1}^{\prime}$ and $\pi_{2}^{\prime}$ are irreducible and inequivalent as boundary representations, then

(1) $\pi$ is irreducible.

(2) Up to equivalence $\left(\iota_{1}, \pi_{1}^{\prime}\right)$ and $\left(\iota_{2}, \pi_{2}^{\prime}\right)$ are the only perfect boundary realizations of $\pi$.

(3) Up to equivalence all boundary realizations of $\pi$ are obtained as

$$
t\left(\iota_{1}, \pi_{1}^{\prime}\right)+(1-t)\left(\iota_{2}, \pi_{2}^{\prime}\right) \quad \text { with } \quad t \in[0,1] .
$$

(4) There exists a dense subspace $\mathcal{H}_{0} \subseteq \mathcal{H}$ and a constant $C_{0}>0$ so that

$$
\lim _{\epsilon \rightarrow 0+} C_{0} \epsilon \sum_{x \in \Gamma} e^{-\epsilon|x| \Gamma}\left\langle v_{1}, \pi(x) v_{2}\right\rangle \overline{\left\langle v_{3}, \pi(x) v_{4}\right\rangle}=\left\langle v_{1}, v_{3}\right\rangle \overline{\left\langle v_{2}, v_{4}\right\rangle}
$$

for $v_{1}, v_{3} \in \mathcal{H}$ and $v_{2}, v_{4} \in \mathcal{H}_{0}$.

\section{Technical Results.}

In this section, let $\Gamma$ be a free group with $A$ as a fixed set of generators and their inverses. Let $(\pi, \mathcal{H})$ be an anisotropic principal series representation of $\Gamma$ and let $\left(\iota_{ \pm}, \pi_{ \pm}^{\prime}\right)$ be the two perfect boundary realizations of $\pi$ described in the introduction. Let $\rho$ be an irreducible unitary representation of $\Gamma$ on a Hilbert space $\mathcal{V}$ of finite dimension $N$. Let $\tau=\pi \otimes \rho$ be the tensor product representation of $\Gamma$ on the Hilbert space $\mathcal{H} \otimes \mathcal{V}$. We will establish that the hypotheses of Theorem 2.9 apply to $\tau$ and its two perfect boundary realizations $\left(\iota_{ \pm} \otimes \mathrm{id}, \tau_{ \pm}^{\prime}\right)$ except in the anomalous case $\sigma=0$ and $\rho \cong \rho \otimes \operatorname{sgn}$. The various conclusions of Theorem 2.9 give Theorems 1.1, 1.2, and 1.6.

Lemma 3.1. Let $T \in \mathcal{B}\left(L^{2}(\Omega, d \nu ; \mathcal{V})\right)$ be a linear bounded $C(\Omega)$-map. Then there exists $t \in L^{\infty}(\Omega, d \nu ; G L(\mathcal{V}))$ such that

$$
(T F)(\omega)=t(\omega) F(\omega) \quad \text { for } F \in L^{2}(\Omega, d \nu ; \mathcal{V})
$$

Proof. We identify $\mathcal{V}$ with $\mathbf{C}^{N}$ and use subscripts on elements of $\mathcal{V}$ to pick. out their coordinates. For every $n=1, \ldots, N$ define $E^{n} \in L^{2}(\Omega, d \nu ; \mathcal{V})$ as follows

$$
\left(E^{n}\right)_{m}(\omega)=\delta(m, n) \equiv \begin{cases}1 & \text { if } m=n \\ 0 & \text { if } m \neq n\end{cases}
$$


Hence for any $F \in C(\Omega ; \mathcal{V})$ we have $F=\sum_{n=1}^{N} \pi_{+}^{\prime}\left(F_{n}\right) E^{n}$. The operator $T$ acts as follows

$$
T F=T\left(\sum_{n=1}^{N} \pi_{+}^{\prime}\left(F_{n}\right) E^{n}\right)=\sum_{n=1}^{N} \pi_{+}^{\prime}\left(F_{n}\right) T E^{n} .
$$

We define

$$
t_{m n}(\omega)=\left(T E^{n}\right)_{m}(\omega) \quad \text { where } t_{m n} \in L^{2}(\Omega, d \nu)
$$

Then

$$
(T F)_{m}(\omega)=\sum_{n=1}^{N} t_{m n}(\omega) F_{n}(\omega)=(t(\omega) F(\omega))_{m}
$$

where $t(\omega) \equiv\left(t_{m n}(\omega)\right)_{N \times N}$ belongs to $L^{2}(\Omega, d \nu ; G L(\mathcal{V}))$. Since $T$ is bounded, it follows from Lusin's Theorem that the entries of $t$ are almost everywhere bounded. Then $t \in L^{\infty}(\Omega, d \nu ; G L(\mathcal{V}))$. Consequently extending by density, we get

$$
(T F)(\omega)=t(\omega) F(\omega) \text { for } F \in L^{2}(\Omega, d \nu ; \mathcal{V})
$$

Endow $G L(\mathcal{V})$ with the norm \|\|$_{\infty}$ obtained by identifying any linear operator $t: \mathcal{V} \longrightarrow \mathcal{V}$ with its matrix $\left(t_{j k}\right)_{j, k}$ and setting $\|t\|_{\infty}=\sup _{j, k}\left|t_{j k}\right|$.

Lemma 3.2. Let $f$ be a function in $L^{1}(\Omega, d \nu ; G L(\mathcal{V}))$. Then

$$
\lim _{n \rightarrow+\infty} \frac{1}{\nu\left(\Omega\left(\omega_{n}\right)\right)} \int_{\Omega\left(\omega_{n}\right)}\left\|f\left(\omega^{\prime}\right)-f(\omega)\right\|_{\infty} d \nu\left(\omega^{\prime}\right)=0
$$

for almost all $\omega=\left(\omega_{0}, \omega_{1}, \ldots, \omega_{n}, \ldots\right) \in \Omega$.

Proof. This is a standard consequence of the boundedness of the maximal function for $L^{1}$-martingales.

Lemma 3.3. If $\gamma \in \Gamma, a \in A$, and $|\gamma a|=|\gamma|+1$, then $\gamma^{-1} \Omega(\gamma a)=\Omega(a)$.

Proof. Thinking of boundary points of $\Omega$ as infinite reduced words, one sees easily that

$$
\gamma^{-1} \Omega(\gamma a) \subseteq \Omega(a) \text { and } \gamma \Omega(a) \subseteq \Omega(\gamma a)
$$

Lemma 3.4. If $\gamma \in \Gamma, a \in A$, and $|\gamma a|=|\gamma|+1$, then $P_{+}(\gamma, \omega)$ is constant for $\omega \in \Omega(\gamma a)$.

Proof. By (1.5)

$$
P_{+}(\gamma, \omega)=\frac{1}{h_{+}(\gamma)} \quad \text { for } \omega \in \Omega(\gamma a)
$$


Lemma 3.5. If $\gamma \in \Gamma, a \in A$, and $|\gamma a|=|\gamma|+1$, then

$$
\frac{d \nu\left(\gamma^{-1} \omega\right)}{d \nu(\omega)}=\frac{\nu(\Omega(a))}{\nu(\Omega(\gamma a))} \quad \text { for } \omega \in \Omega(\gamma a)
$$

Proof. From (1.6) we have that

$$
\frac{d \nu\left(\gamma^{-1} \omega\right)}{d \nu(\omega)}=\left|P_{+}(\gamma, \omega)\right|^{2}
$$

By Lemma 3.4 above, $\left|P_{+}(\gamma, \omega)\right|^{2}$ is constant for $\omega \in \Omega(\gamma a)$. Clearly this constant also gives

$$
\frac{\nu\left(\gamma^{-1} \Omega(\gamma a)\right)}{\nu(\Omega(\gamma a))}=\frac{\nu(\Omega(a))}{\nu(\Omega(\gamma a))} .
$$

Lemma 3.6. Let $f$ be a function in $L^{\infty}(\Omega, d \nu ; G L(\mathcal{V}))$. Let $\rho$ be a unitary representation of $\Gamma$ on a finite dimensional Hilbert space $\mathcal{V}$ and let $Q: \Gamma \times$ $\Omega \longrightarrow \mathbf{C}$ be a function, $\nu$-measurable in $\omega$, such that

(1) $|Q(x, \omega)|=1$ for all $x \in \Gamma$ and $\omega \in \Omega$.

(2) $f\left(x^{-1} \omega\right)=Q(x, \omega) \rho\left(x^{-1}\right) f(\omega) \rho(x)$ for all $x \in \Gamma$ and $\omega \in \Omega$.

(3) If $\gamma, y \in \Gamma$ and $\gamma \notin \Gamma(y)$ then $Q(\gamma, \omega)$ is constant for $\omega \in \Omega(y)$. Then there exists $a \in A$ and $t_{0} \in G L(\mathcal{V})$ such that

$$
f(\omega)=t_{0} \quad \text { for almost all } \omega \in \Omega(a) .
$$

Proof. By Lemma 3.2 there exists $\omega=\left(\omega_{0}, \omega_{1}, \ldots, \omega_{n}, \ldots\right) \in \Omega$ such that

$$
\lim _{n \rightarrow+\infty} \frac{1}{\nu\left(\Omega\left(\omega_{n}\right)\right)} \int_{\Omega\left(\omega_{n}\right)}\left\|f\left(\omega^{\prime}\right)-s\right\|_{\infty} d \nu\left(\omega^{\prime}\right)=0
$$

where $s=f(\omega)$. For every $n \geq 1$ let $a_{n}=\gamma_{n}^{-1} \omega_{n}$ where $\gamma_{n}=\omega_{n-1}$. According to (3) $Q\left(\gamma_{n}, \omega^{\prime}\right) s$ has a constant value for $\omega^{\prime} \in \Omega\left(\omega_{n}\right)$. Let $s_{n}=Q\left(\gamma_{n}, \omega^{\prime}\right) s$ be that constant value. Then

$$
\begin{aligned}
& \frac{1}{\nu\left(\Omega\left(a_{n}\right)\right)} \int_{\Omega\left(a_{n}\right)}\left\|\rho\left(\gamma_{n}\right) f\left(\omega^{\prime}\right) \rho\left(\gamma_{n}^{-1}\right)-s_{n}\right\|_{\infty} d \nu\left(\omega^{\prime}\right) \\
& \quad=\frac{\nu\left(\Omega\left(\omega_{n}\right)\right)}{\nu\left(\Omega\left(a_{n}\right)\right)} \frac{1}{\nu\left(\Omega\left(\omega_{n}\right)\right)} \int_{\gamma_{n} \Omega\left(a_{n}\right)}\left\|\rho\left(\gamma_{n}\right) f\left(\gamma_{n}^{-1} \omega^{\prime}\right) \rho\left(\gamma_{n}^{-1}\right)-s_{n}\right\|_{\infty} d \nu\left(\gamma_{n}^{-1} \omega^{\prime}\right) \\
& =\frac{1}{\nu\left(\Omega\left(\omega_{n}\right)\right)} \int_{\Omega\left(\omega_{n}\right)}\left\|f\left(\omega^{\prime}\right)-s\right\|_{\infty} d \nu\left(\omega^{\prime}\right) \quad(\text { using Lemmas 3.3 and 3.5). }
\end{aligned}
$$


Hence

$$
\lim _{n \rightarrow+\infty} \frac{1}{\nu\left(\Omega\left(a_{n}\right)\right)} \int_{\Omega\left(a_{n}\right)}\left\|\rho\left(\gamma_{n}\right) f\left(\omega^{\prime}\right) \rho\left(\gamma_{n}^{-1}\right)-s_{n}\right\|_{\infty} d \nu\left(\omega^{\prime}\right)=0 .
$$

Since $\mathcal{U}(\mathcal{V})$ is compact, since $s_{n} \in\{\alpha s ;|\alpha|=1\}$, and since $A$ is finite, we may select a sequence $\left(n_{j}\right)$ so that $\rho\left(\gamma_{n_{j}}\right) \rightarrow \rho_{0}$ for some $\rho_{0}$ in $\mathcal{U}(\mathcal{V})$, so that $s_{n_{j}} \rightarrow t$ for some $t \in G L(\mathcal{V})$, and so that $a_{n_{j}}=a$ for all $j$ and for some $a \in A$. Then

$$
\int_{\Omega(a)}\left\|\rho_{0} f\left(\omega^{\prime}\right) \rho_{0}^{-1}-t\right\|_{\infty} d \nu\left(\omega^{\prime}\right)=0
$$

That is

$$
f(\omega)=t_{0} \quad \text { for almost all } \omega \in \Omega(a)
$$

where $t_{0} \equiv \rho_{0}^{-1} t \rho_{0}$.

In analogy with $\Gamma(y)$, the set of all words which start with the letters of $y$, define $\tilde{\Gamma}(y)=\Gamma\left(y^{-1}\right)^{-1}$, the set of all words that end with the letters of $y$.

Lemma 3.7. Let $(\Gamma, A)$ be a free group. Let $\rho$ be a unitary representation of $\Gamma$ on a finite dimensional Hilbert space $\mathcal{V}$ and let $q: \Gamma \longrightarrow \mathbf{C}$ be a multiplicative function (not a character, see (1.1)). If there exists $a \in A$ and $t_{0} \in G L(\mathcal{V})$ such that

$$
t_{0} \rho(x)=q(x) \rho(x) t_{0} \quad \text { for } x \in \Gamma(a) \backslash \tilde{\Gamma}\left(a^{-1}\right)
$$

then

$$
t_{0} \rho(x)=q(x) \rho(x) t_{0} \quad \text { for } x \in \Gamma
$$

Proof. Fix $x \in \Gamma\left(a_{1}\right) \cap \tilde{\Gamma}\left(a_{2}\right)$ where $a_{1}, a_{2} \in A$ and $a_{2} \neq a^{-1}$. Choose $w_{1} \in \Gamma(a) \operatorname{such}$ that $w_{1} \notin \tilde{\Gamma}\left(a^{-1}\right)$ and $w_{1} \notin \tilde{\Gamma}\left(a_{1}^{-1}\right)$. Choose $w_{2} \in \Gamma(a)$ such that $w_{2} \notin \tilde{\Gamma}\left(a^{-1}\right)$. Then $w_{1}, w_{2}, w_{1} x w_{2} \in \Gamma(a) \backslash \tilde{\Gamma}\left(a^{-1}\right)$, and $\left|w_{1} x w_{2}\right|=$ $\left|w_{1}\right|+|x|+\left|w_{2}\right|$. Hence

$$
\begin{aligned}
& t_{0} \rho\left(w_{1} x w_{2}\right)=q\left(w_{1} x w_{2}\right) \rho\left(w_{1} x w_{2}\right) t_{0} \\
& \quad \Rightarrow q\left(w_{1}\right) \rho\left(w_{1}\right) t_{0} \rho(x) \rho\left(w_{2}\right)=q\left(w_{1}\right) \rho\left(w_{1}\right) q\left(x w_{2}\right) \rho\left(x w_{2}\right) t_{0} \\
& \quad \Rightarrow t_{0} \rho(x) \rho\left(w_{2}\right)=q(x) \rho(x) q\left(w_{2}\right) \rho\left(w_{2}\right) t_{0}=q(x) \rho(x) t_{0} \rho\left(w_{2}\right) \\
& \quad \Rightarrow t_{0} \rho(x)=q(x) \rho(x) t_{0} .
\end{aligned}
$$

For $x \in \tilde{\Gamma}\left(a^{-1}\right)$ choose $b \in A$ such that $b \neq a$ and $b \neq a^{-1}$. Then $b$ and $x b$ are words which don't end with $a^{-1}$ and $|x b|=|x|+1$. In this case we already know that

$$
t_{0} \rho(x b)=q(x b) \rho(x b) t_{0} \quad \text { and } \quad t_{0} \rho(b)=q(b) \rho(b) t_{0} .
$$


Hence

$$
t_{0} \rho(x) \rho(b)=q(x) \rho(x) q(b) \rho(b) t_{0}=q(x) \rho(x) t_{0} \rho(b) .
$$

Recall that $\sigma \in \operatorname{Spec}(\mu)$ is not allowed to be an endpoint. We define

$$
q(x)=\frac{h_{+}(x)}{h_{-}(x)} \quad \text { for } x \in \Gamma .
$$

This makes $q$ a multiplicative function of modulus one on $\Gamma$.

Lemma 3.8. If $q^{2}(a)=1$ for all $a \in A$, then $\sigma=0$ and $q(x)=\operatorname{sgn}(x) \equiv$ $(-1)^{|x|}$ for all $x \in \Gamma$.

Proof. From [Figà-Talamanca-Steger], Proposition 1.3.4, we will use

(1) $2 w_{+}=\mu(c)\left(h_{+}^{-1}(c)-h_{+}(c)\right)$ for any $c \in A$ and

(2) $2 w_{+}=\sigma-\sum_{b \in A} \mu(b) h_{+}(b)$.

We proceed, dividing the proof into two cases.

(A) There is a $c \in A$ such that $h_{+}(c) \in \mathbf{R}$. By (1) we note first that $w_{+}$ is real and then that $h_{+}(b)$ is real for all $b \in A$. By [Figà-TalamancaSteger], Lemma 2.3.1, and the discussion thereafter, we know that $h_{+}=h_{-}$ and $w_{+}=w_{-}$is impossible for $\sigma$ in the interior of $\operatorname{Spec}(\mu)$. This eliminates case $(\mathrm{A})$.

(B) For all $a \in A$ we have $h_{+}(a) \in i \mathbf{R}$. Since $h_{+}(a) \in i \mathbf{R}$ for all $a \in A$, $w_{+} \in i \mathbf{R}$. By (2) $\sigma=0$. We also have that $q(a)=-1$ for all $a \in A$, so $q(x)=(-1)^{|x|}$ for all $x \in \Gamma$.

Proposition 3.9. The two boundary representations $\tau_{ \pm}^{\prime}$ are irreducible.

Proof. We only need to work with $\tau_{+}^{\prime}$. Let $\mathcal{H}_{1}$ be a closed $(\Gamma, C(\Omega))$-stable subspace in $L^{2}(\Omega, d \nu ; \mathcal{V})$. Then $\mathcal{H}_{2}=\mathcal{H}_{1}^{\perp}$ has the same property, so the orthogonal projection $T: L^{2}(\Omega, d \nu ; \mathcal{V}) \longrightarrow L^{2}(\Omega, d \nu ; \mathcal{V})$ onto $\mathcal{H}_{1}$ commutes with $\tau_{+}^{\prime}(x)$ and $\tau_{+}^{\prime}(G)$ for all $x \in \Gamma$ and $G \in C(\Omega)$. So, as usual, we only need to prove that any bounded $(\Gamma, C(\Omega))$-map $T$ on $L^{2}(\Omega, d \nu ; \mathcal{V})$ is scalar. Applying Lemma 3.1 there exists $t \in L^{\infty}(\Omega, d \nu ; G L(\mathcal{V}))$ such that

$$
(T F)(\omega)=t(\omega) F(\omega) \text { for } F \in L^{2}(\Omega, d \nu ; \mathcal{V}) .
$$

On the other hand, for all $x \in \Gamma$ and $F \in L^{2}(\Omega, d \nu ; \mathcal{V})$

$$
\left(\tau_{+}^{\prime}(x) T F\right)(\omega)=P_{+}(x, \omega) \rho(x) t\left(x^{-1} \omega\right) F\left(x^{-1} \omega\right)
$$

and

$$
\left(T \tau_{+}^{\prime}(x) F\right)(\omega)=t(\omega) P_{+}(x, \omega) \rho(x) F\left(x^{-1} \omega\right)
$$




\section{Therefore}

$$
t\left(x^{-1} \omega\right)=\rho\left(x^{-1}\right) t(\omega) \rho(x) \quad \text { for any } x \in \Gamma .
$$

From Lemma 3.6, with $Q(x, \omega)=1$ there exists $a \in \Gamma$ and $t_{0} \in G L(\mathcal{V})$ such that $t(\omega)=t_{0}$ for almost all $\omega \in \Omega(a)$. Fix $x \in \Gamma(a) \backslash \tilde{\Gamma}\left(a^{-1}\right)$. Then $x \omega \in \Omega(a)$ and the product $x \omega$ does not simplify when $\omega \in \Omega(a)$. Therefore

$$
t_{0}=t(x \omega)=\rho(x) t(\omega) \rho\left(x^{-1}\right)=\rho(x) t_{0} \rho\left(x^{-1}\right)
$$

for almost all $\omega \in \Omega(a)$. By Lemma 3.7, with $q(x)=1$, it follows that

$$
t_{0} \rho(x)=\rho(x) t_{0} \quad \text { for } x \in \Gamma .
$$

Then $t_{0}$ commutes with $\rho(x)$ for all $x \in \Gamma$. As a consequence of Schur's Lemma, $t_{0}$ is scalar. That is, there exists $\alpha \in \mathbf{C}$ such that

$$
t(\omega)=\alpha \operatorname{id}_{\mathcal{V}} \quad \text { for almost all } \omega \in \Omega(a) .
$$

We want now to extend this result to $\Omega$. Fix $b \in A$. Find $z \in \Gamma(a) \cap \tilde{\Gamma}(b)$. Then for $\omega \notin \Omega\left(b^{-1}\right), z \omega \in \Omega(a)$. Thus $t(\omega)=\alpha$ id $_{\mathcal{V}}$ almost everywhere on $\Omega \backslash \Omega\left(b^{-1}\right)$. Vary $b$ to see that this holds almost everywhere on $\Omega$. Thus $T$ is multiplication by $\alpha$.

Proposition 3.10. Excluding the anomalous case that $\sigma=0$ and $\rho \cong$ $\rho \otimes \operatorname{sgn}$, the two boundary representations $\tau_{ \pm}^{\prime}$ are inequivalent.

Proof. We proceed by contradiction. Suppose that

$$
T: L^{2}(\Omega, d \nu ; \mathcal{V}) \longrightarrow L^{2}(\Omega, d \nu ; \mathcal{V})
$$

is a unitary $(\Gamma, C(\Omega))$-map that intertwines $\tau_{+}^{\prime}(x)$ with $\tau_{-}^{\prime}(x)$ and $\tau_{+}^{\prime}(G)$ with $\tau_{-}^{\prime}(G)$ for any $x$ in $\Gamma$ and $G$ in $C(\Omega)$. By Lemma 3.1 there exists $t \in L^{\infty}(\Omega, d \nu ; G L(\mathcal{V}))$ such that

$$
(T F)(\omega)=t(\omega) F(\omega) \quad \text { for } F \in L^{2}(\Omega, d \nu ; \mathcal{V})
$$

For all $x \in \Gamma$ and $F \in L^{2}(\Omega, d \nu ; \mathcal{V})$ the following holds.

$$
\left(\tau_{-}^{\prime}(x) T F\right)(\omega)=P_{-}(x, \omega) \rho(x) t\left(x^{-1} \omega\right) F\left(x^{-1} \omega\right)
$$

and

$$
\left(T \tau_{+}^{\prime}(x) F\right)(\omega)=t(\omega) P_{+}(x, \omega) \rho(x) F\left(x^{-1} \omega\right)
$$

Hence

$$
t\left(x^{-1} \omega\right)=\frac{P_{+}(x, \omega)}{P_{-}(x, \omega)} \rho\left(x^{-1}\right) t(\omega) \rho(x) .
$$


By Lemma 3.6 with

$$
Q(x, \omega)=\frac{P_{+}(x, \omega)}{P_{-}(x, \omega)}=\frac{P_{+}(x, \omega)}{P_{+}(x, \omega)^{-}},
$$

there exists $a \in \Gamma$ and $t_{0} \in G L(\mathcal{V})$ such that

$$
t(\omega)=t_{0} \quad \text { for almost all } \omega \in \Omega(a) .
$$

Fix $x \in \Gamma(a) \backslash \tilde{\Gamma}\left(a^{-1}\right)$. When $\omega \in \Omega(a)$, the product $x \omega$ does not simplify and $x \omega \in \Omega(a)$. By the absence of cancellation

$$
Q\left(x^{-1}, \omega\right)=\frac{h_{+}\left(x^{-1}\right)}{h_{-}\left(x^{-1}\right)}=\frac{h_{+}(x)}{h_{-}(x)}=q(x)
$$

for $\omega \in \Omega(a)$. For almost every $\omega \in \Omega(a)$

$$
t_{0}=t(x \omega)=Q\left(x^{-1}, \omega\right) \rho(x) t(\omega) \rho\left(x^{-1}\right)=q(x) \rho(x) t_{0} \rho\left(x^{-1}\right) .
$$

Thus, by Lemma 3.7, the relation $t_{0} \rho(x)=q(x) \rho(x) t_{0}$ holds for all $x \in \Gamma$. For $x, y \in \Gamma$

$$
\begin{aligned}
q(x y) \rho(x y) t_{0} & =t_{0} \rho(x y)=t_{0} \rho(x) \rho(y) \\
& =q(x) \rho(x) t_{0} \rho(y)=q(x) \rho(x) q(y) \rho(y) t_{0}=q(x) q(y) \rho(x y) t_{0} .
\end{aligned}
$$

Hence

$$
q(x) q(y)=q(x y) \quad \text { for } x, y \in \Gamma .
$$

This means that $q$ is a character of $\Gamma$. Then for any $a \in A$ we have

$$
1=q(e)=q\left(a^{-1} a\right)=q\left(a^{-1}\right) q(a) .
$$

That is

$$
q^{2}(a)=1 \quad \text { for } a \in A
$$

By Lemma 3.8 it follows that $\sigma=0$ and $q(x)=\operatorname{sgn}(x)$ for all $x \in \Gamma$. Since $t_{0} \rho(x)=\operatorname{sgn}(x) \rho(x) t_{0}, t_{0}$ is an intertwiner between $\rho$ and $\rho \otimes \operatorname{sgn}$. Since the anomalous case is excluded, and since $\sigma=0, \rho$ and $\rho \otimes \operatorname{sgn}$ cannot be equivalent. Therefore $t_{0}$ must be 0 .

Proposition 3.11. The two boundary realizations $\left(\iota_{ \pm} \otimes \mathrm{id}, \tau_{ \pm}^{\prime}\right)$ satisfy the $(F T C)$.

Proof. Given Lemma 2.8, we need only to work with $\left(\iota_{ \pm}, \pi_{ \pm}^{\prime}\right)$. Remember that $\iota_{ \pm}$are unitary, and that the Hilbert-Schmidt norm is invariant under unitary operator action on either side. Observe also that for any 
$a \in A$ the functions $\mathbf{1}_{\Omega(a)}$ and $\mathbf{1}-\mathbf{1}_{\Omega(a)}$ are idempotent, so $\pi_{+}^{\prime}\left(\mathbf{1}_{\Omega(a)}\right)$ and $\pi_{-}^{\prime}\left(\mathbf{1}-\mathbf{1}_{\Omega(a)}\right)$ are projections and consequently the operators $\iota_{+}^{*} \pi_{+}^{\prime}\left(\mathbf{1}_{\Omega(a)}\right) \iota_{+}$ and $\iota_{-}^{*} \pi_{-}^{\prime}\left(\mathbf{1}-\mathbf{1}_{\Omega(a)}\right) \iota_{-}$are too. Recall that $J=\iota_{-} \iota_{+}^{*}$ intertwines

$$
\left(\pi_{+}^{\prime}(x), L^{2}(\Omega, d \nu)\right) \quad \text { with } \quad\left(\pi_{-}^{\prime}(x), L^{2}(\Omega, d \nu)\right) .
$$

Therefore, our problem reduces as follows

$$
\begin{gathered}
\left(\iota_{+}^{*} \pi_{+}^{\prime}\left(\mathbf{1}_{\Omega(a)}\right) \iota_{+}, \iota_{-}^{*} \pi_{-}^{\prime}\left(\mathbf{1}-\mathbf{1}_{\Omega(a)}\right) \iota_{-}\right)_{B^{+}(\mathcal{H})} \\
=\left\|\iota_{+}^{*} \pi_{+}^{\prime}\left(\mathbf{1}_{\Omega(a)}\right) \iota_{+} \iota_{-}^{*} \pi_{-}^{\prime}\left(\mathbf{1}-\mathbf{1}_{\Omega(a)}\right) \iota_{-}\right\|_{H S}^{2} \\
=\left\|\pi_{+}^{\prime}\left(\mathbf{1}_{\Omega(a)}\right) J^{*} \pi_{-}^{\prime}\left(\mathbf{1}-\mathbf{1}_{\Omega(a)}\right)\right\|_{H S}^{2} .
\end{gathered}
$$

Section 3 of [Figà-Talamanca-Steger] gives the following description of $J$. Define a set $\left(F_{x}\right)_{x \in \Gamma}$ of functions in $\mathcal{K}(\Omega)$, the space of locally constant functions in $L^{2}(\Omega, d \nu)$, by

$$
\begin{aligned}
F_{e} & =\mathbf{1}_{\Omega} \\
F_{x a} & =\frac{1}{\nu(\Omega(a))} \mathbf{1}_{\Omega(a x)}-\frac{1}{\nu(\Omega(x))} \mathbf{1}_{\Omega(x)} \quad \text { for }|a|=1 \text { and }|x a|=|x|+1 .
\end{aligned}
$$

We know that $\left(F_{x}\right)_{x \in \Gamma}$ spans $\mathcal{K}(\Omega)$ and

$$
\begin{aligned}
J F_{e} & =F_{e} \\
J F_{x a} & =\frac{w_{-}}{w_{+}} \frac{h_{+}(x)}{h_{-}(x)} F_{x a} \text { for }|a|=1 \text { and }|x a|=|x|+1 .
\end{aligned}
$$

For $x \in \Gamma$ let $\mathcal{K}_{x}$ be the linear span of $\left\{F_{x a} ;|x a|=|x|+1\right.$ and $\left.a \in A\right\}$. This means that

$$
\mathcal{K}_{x}=\left\{F \in \mathcal{K}(\Omega) ; \operatorname{supp}(F) \subseteq \Omega(x), \quad \int_{\Omega} F(\omega) d \nu(\omega)=0,\right.
$$

and $F$ depends only on the first $|x|+1$ letters of $\omega\}$.

Define $\mathcal{K}_{0}=\mathbf{C} \cdot \mathbf{1}_{\Omega}$. Then the spaces $\mathcal{K}_{x}$ together with $\mathcal{K}_{0}$ span $\mathcal{K}(\Omega)$. Each element of $\mathcal{K}_{x}$ is an eigenvector of $J$. In addition, for $x \neq y, \mathcal{K}_{x}$ and $\mathcal{K}_{y}$ are orthogonal to each other and to $\mathcal{K}_{0}$, with respect to integration by $\nu$. For these reasons, we can write

$$
\begin{aligned}
L^{2}(\Omega, d \nu) & \cong \mathcal{K}_{0} \oplus \bigoplus_{x \in \Gamma} \mathcal{K}_{x} \\
\left.J\right|_{\mathcal{K}_{0}} & =\operatorname{id}_{\mathcal{K}_{0}} \\
\left.J\right|_{\mathcal{K}_{x}} & =\frac{w_{-}}{w_{+}} \frac{h_{+}(x)}{h_{-}(x)} \operatorname{id}_{\mathcal{K}_{x}} .
\end{aligned}
$$


Let $x \in \Gamma$ and $|x| \geq 1$. If $x \in \Omega(a)$ then $\pi_{-}^{\prime}\left(\mathbf{1}-\mathbf{1}_{\Omega(a)}\right)\left(\mathcal{K}_{x}\right)=0$. If $x \notin \Omega(a)$ we have

$$
\pi_{+}^{\prime}\left(\mathbf{1}_{\Omega(a)}\right) J^{*} \pi_{-}^{\prime}\left(\mathbf{1}-\mathbf{1}_{\Omega(a)}\right)\left(\mathcal{K}_{x}\right) \subseteq \pi_{+}^{\prime}\left(\mathbf{1}_{\Omega(a)}\right)\left(\mathcal{K}_{x}\right)=0 .
$$

Hence $\pi_{+}^{\prime}\left(\mathbf{1}_{\Omega(a)}\right) J^{*} \pi_{-}^{\prime}\left(\mathbf{1}-\mathbf{1}_{\Omega(a)}\right)$ is identically zero on $\bigoplus_{|x| \geq 1} \mathcal{K}_{x}$. Therefore to compute the Hilbert-Schmidt norm, we need only to work with the restriction to $\mathcal{K}_{0} \oplus \mathcal{K}_{e}$. Since $\mathcal{K}_{0} \oplus \mathcal{K}_{e}$ is finite dimensional, the Hilbert-Schmidt norm is finite.

\section{Anomalous case.}

Let $\Gamma$ be a free group and let $A$ be a set of generators and inverses for $\Gamma$. As in the introduction, fix a symmetric probability measure $\mu$ on $A$. The anisotropic principal series representation $\pi$ is constructed from the pair $(\mu, \sigma)$ where $\sigma$ is in the interior of $\operatorname{Spec}(\mu)$. The burden of this section is to explain the special case $\sigma=0$.

Since the function sgn is a character

$$
\left(\left(f_{1}\right) \operatorname{sgn}\right) *\left(\left(f_{2}\right) \operatorname{sgn}\right)=\left(f_{1} * f_{2}\right) \operatorname{sgn}
$$

where $f_{1}$ and $f_{2}$ are functions on $\Gamma$ and "*" denotes convolution. The symbols in the following lemma are from (1.2).

Lemma 4.1. When $\sigma=0$

(1) $g_{+}=-\left(g_{-}\right) \operatorname{sgn}$

(2) $w_{+}=-w_{-}$

(3) $h_{+}=\left(h_{-}\right) \operatorname{sgn}$

(4) $w_{ \pm}, h_{ \pm}(a) \in i \mathbf{R}$ for $a \in A$

(5) $\phi(x)=0$ if $|x|$ is odd.

Proof. For $\gamma \notin \operatorname{Spec}(\mu)$ recall that $g_{\gamma}$ is defined as the inverse of $\gamma \delta_{e}-\mu$, both functions acting on $\ell^{2}(\Gamma)$ by left convolution. From (4.1)

$$
-g_{-\gamma}=\left(\gamma \delta_{e}+\mu\right)^{-1}=\left(\left(\gamma \delta_{e}-\mu\right) \operatorname{sgn}\right)^{-1}=\left(\gamma \delta_{e}-\mu\right)^{-1} \operatorname{sgn}=\left(g_{\gamma}\right) \operatorname{sgn}
$$

where multiplication by sgn is pointwise multiplication but all inverses are inverses for the convolution algebra. (1) then follows from the definition of $g_{ \pm}$ as the limit $g_{0 \pm i 0}$. (2) and (3) follow from the equality $g_{ \pm}=h_{ \pm} /\left(2 w_{ \pm}\right)$given that $h_{ \pm}$is a multiplicative function and $w_{ \pm}$is a constant. Since $w_{+}=\bar{w}_{-}$ and $h_{+}=\bar{h}_{-},(4)$ is a consequence of (2) and (3). (5) is immediate from (1) and the definition $\phi=\left(g_{+}-g_{-}\right) /\left(g_{+}(e)-g_{-}(e)\right)$. 
Since $(\pi, \mathcal{H})$ is the result of the Gel'fand-Raikov construction applied to $\phi$, there is a cyclic vector $k_{0} \in \mathcal{H}$ such that

$$
\left\langle k_{0}, \pi(x) k_{0}\right\rangle=\phi(x) \quad \text { for } x \in \Gamma .
$$

Let

$$
\begin{aligned}
& \mathcal{H}_{e}=\text { closed-span }\left\{\pi(x) k_{0} ; x \in \Gamma,|x| \text { is even }\right\} \\
& \mathcal{H}_{o}=\text { closed-span }\left\{\pi(y) k_{0} ; y \in \Gamma,|y| \text { is odd }\right\}
\end{aligned}
$$

By (5) of Lemma 4.1, these two spaces are orthogonal to one another, so $\mathcal{H}=\mathcal{H}_{e} \oplus \mathcal{H}_{o}$. Define $S: \mathcal{H} \rightarrow \mathcal{H}$ by

$$
\left.S\right|_{\mathcal{H}_{e}}=\left.\operatorname{id}_{\mathcal{H}_{e}} \quad S\right|_{\mathcal{H}_{o}}=-\mathrm{id}_{\mathcal{H}_{o}} .
$$

\section{Lemma 4.2.}

(1) $S \pi(x)^{\circ}=\operatorname{sgn}(x) \pi(x) S$ for $x \in \Gamma$.

(2) $\pi_{+}^{\prime}(x)=\operatorname{sgn}(x) \pi_{-}^{\prime}(x)$ for $x \in \Gamma$.

(3) $\iota_{+} S=\iota_{-}$.

Proof. (1) follows from the definitions. To establish (2), it is enough to do so for $x \in A$, and that is immediate by the definition (1.4) of $\pi_{ \pm}^{\prime}$ and by (3) of Lemma 4.1. Since $\iota_{+} S k_{0}=1=\iota_{-} k_{0}$,

$$
\begin{aligned}
\iota_{+} S \pi(x) k_{0} & =\operatorname{sgn}(x) \iota_{+} \pi(x) S k_{0}=\operatorname{sgn}(x) \pi_{+}^{\prime}(x) \iota_{+} S k_{0} \\
& =\pi_{-}^{\prime}(x) \iota_{-} k_{0}=\iota_{-} \pi(x) k_{0}
\end{aligned}
$$

for $x \in \Gamma$. Since the $\Gamma$-translates of $k_{0}$ are dense in $\mathcal{H}$, this proves (3).

Fix $\rho$ an irreducible unitary representation of $\Gamma$ on a finite dimensional Hilbert space $\mathcal{V}$, such that $\rho \cong \rho \otimes$ sgn. Then there exists a unitary map $s: \mathcal{V} \longrightarrow \mathcal{V}$ such that

$$
s \rho(x)=\operatorname{sgn}(x) \rho(x) s .
$$

Observe that since $s^{2} \rho(x)=\rho(x) s^{2}, s^{2}$ is a scalar operator. Multiplying $s$ by an opportune constant, we may assume $s^{2}$ is the identity operator.

We consider, the operator $S \otimes s: \mathcal{H} \otimes \mathcal{V} \longrightarrow \mathcal{H} \otimes \mathcal{V}$.

\section{Lemma 4.3.}

(1) $(S \otimes s) \tau(x)=\tau(x)(S \otimes s)$ for all $x \in \Gamma$.

(2) $(S \otimes s)^{2}=\mathrm{id}_{\mathcal{H} \otimes \mathcal{V}}$.

(3) $S \otimes s$ is unitary and self adjoint. 


$$
\begin{array}{ll}
\text { (4) } & \tau_{+}^{\prime}(x)=\operatorname{sgn}(x) \tau_{-}^{\prime}(x) \text { for all } x \in \Gamma . \\
\text { (5) } \quad\left(\iota_{+} \otimes \mathrm{id}\right)(S \otimes s)=\left(\iota_{-} \otimes s\right) .
\end{array}
$$

Proof. (1) follows from (4.3) and (1) of Lemma 4.2. In fact

$$
\begin{aligned}
(S \otimes s) \tau(x) & =(S \otimes s)(\pi(x) \otimes \rho(x))=(S \pi(x) \otimes s \rho(x)) \\
& =(\operatorname{sgn}(x) \pi(x) S \otimes \operatorname{sgn}(x) \rho(x) s)=\tau(x)(S \otimes s) .
\end{aligned}
$$

For $(2)(S \otimes s)^{2}=\left(S^{2} \otimes s^{2}\right)=\mathrm{id}_{\mathcal{H}} \otimes \mathrm{id}_{\mathcal{V}}=\mathrm{id}_{\mathcal{H} \otimes \mathcal{V}}$. Remember that $S \otimes s$ is the tensor product of unitary self adjoint operators. This gives (3). By (2) of Lemma $4.2 \pi_{+}^{\prime}(x)=\operatorname{sgn}(x) \pi_{-}^{\prime}(x)$ for any $x \in \Gamma$, and so $\tau_{+}^{\prime}(x)=\pi_{+}^{\prime}(x) \otimes \rho(x)$ $=\operatorname{sgn}(x) \pi_{-}^{\prime}(x) \otimes \rho(x)=\operatorname{sgn}(x) \tau_{-}^{\prime}(x)$, proving (4). Finally, (3) of Lemma 4.2 gives (5).

Let $\mathcal{V}_{e}$ and $\mathcal{V}_{o}$ be the 1 and -1 eigenspaces of $s$ respectively. This means that

$$
\left.s\right|_{\mathcal{V}_{e}}=\left.\left.\mathrm{id}\right|_{\mathcal{V}_{e}} \quad s\right|_{\mathcal{V}_{o}}=-\left.\mathrm{id}\right|_{\mathcal{V}_{o}} \quad \mathcal{V}=\mathcal{V}_{e} \oplus \mathcal{V}_{o}
$$

Then the 1 and -1 eigenspaces of $S \otimes s$ are

$$
\mathcal{W}_{e}=\left(\mathcal{H}_{e} \otimes \mathcal{V}_{e}\right) \oplus\left(\mathcal{H}_{o} \otimes \mathcal{V}_{o}\right), \quad \mathcal{W}_{o}=\left(\mathcal{H}_{e} \otimes \mathcal{V}_{o}\right) \oplus\left(\mathcal{H}_{o} \otimes \mathcal{V}_{e}\right)
$$

Let $\tau_{e}$ and $\tau_{o}$ be the representations of $\Gamma$ on $\mathcal{W}_{e}$ and $\mathcal{W}_{o}$ respectively. From the perfect boundary realization $\left(\iota_{+} \otimes \mathrm{id}, \tau_{+}^{\prime}\right)$ of $\tau$ construct boundary realizations, no longer perfect, $\left(\iota_{e}, \tau_{+}^{\prime}\right)$ and $\left(\iota_{o}, \tau_{+}^{\prime}\right)$ of $\tau_{e}$ and $\tau_{o}$ respectively, by restricting $\iota_{+} \otimes \mathrm{id}$ to $\mathcal{W}_{e}$ and $\mathcal{W}_{o}$. Condition (3) in Definition 1.4 holds on account of Proposition 3.9, which says that $\tau_{+}^{\prime}$ is irreducible as a $(\Gamma, C(\Omega)$ )representation.

The following result from [Steger] is parallel to Theorem 2.9.

Theorem 4.4. Let $\Gamma$ be a free group, let $A$ be some set of generators and their inverses for $\Gamma$, and define length on $\Gamma$ in terms of $A$. Let $\left(\pi_{1}, \mathcal{H}_{1}\right)$ be an arbitrary unitary representation of $\Gamma$ and let $\left(\iota_{1}, \pi^{\prime}\right)$ be a boundary realization of $\pi_{1}$. Suppose that $\left(\iota_{1}, \pi^{\prime}\right)$ is not perfect and that it satisfies the $(F T C)$ with itself. Suppose further that $\pi^{\prime}$ is irreducible as a $(\Gamma, C(\Omega))$-representation. Then

(1) $\pi_{1}$ is irreducible.

(2) Up to equivalence, $\left(\iota_{1}, \pi^{\prime}\right)$ is the only boundary realization of $\pi_{1}$.

(3) There is a dense subspace $\mathcal{H}_{01} \subseteq \mathcal{H}_{1}$ and a constant $C_{0}>0$ so that

$$
\lim _{\epsilon \rightarrow 0+} C_{0} \epsilon \sum_{x \in \Gamma} e^{-\epsilon|x|_{\Gamma}}\left\langle v_{1}, \pi(x) v_{2}\right\rangle \overline{\left\langle v_{3}, \pi(x) v_{4}\right\rangle}=\left\langle v_{1}, v_{3}\right\rangle \overline{\left\langle v_{2}, v_{4}\right\rangle}
$$

for $v_{1}, v_{3} \in \mathcal{H}_{1}$ and $v_{2}, v_{4} \in \mathcal{H}_{01}$. 
The rest of this section is devoted to showing that the abstract Theorem 4.4 applies to our particular situation.

Theorem 4.5. Let $\sigma=0$ and $\rho \cong \rho \otimes \operatorname{sgn}$. Let $\tau_{e}$ and $\tau_{o}$ be the two direct summands of $\tau$ defined previously. The conclusions of Theorem 4.4 apply to $\tau_{e}$ and its realization $\left(\iota_{e}, \tau_{+}^{\prime}\right)$ and also to $\tau_{o}$ and its realization $\left(\iota_{o}, \tau_{+}^{\prime}\right)$.

Accordingly we have $\tau_{+}^{\prime} \cong \tau_{e} \oplus \tau_{o}$ as representations of $\Gamma$, where $\tau_{+}^{\prime}$ is realized perfectly on the boundary, but neither of its two irreducible summands can be realized except in conjunction with the other.

Neither $\left(\iota_{e}, \tau_{+}^{\prime}\right)$ nor $\left(\iota_{o}, \tau_{+}^{\prime}\right)$ is a perfect realization, verifying one of the hypotheses of Theorem 4.4. Proposition 3.9 asserts that $\tau_{+}^{\prime}$ is an irreducible $(\Gamma, C(\Omega))$-representation, verifying another. It remains only to show that $\left(\iota_{e}, \tau_{+}^{\prime}\right)$ satisfies the (FTC) with itself as a realization of $\tau_{e}$ and similarly for $\left(\iota_{o}, \tau_{+}^{\prime}\right)$. This can be reduced to Proposition 3.11 , which says that $\left(\iota_{+} \otimes \mathrm{id}, \tau_{+}^{\prime}\right)$ and $\left(\iota_{-} \otimes \mathrm{id}, \tau_{-}^{\prime}\right)$ satisfy the (FTC) as realizations of $\tau$.

Lemma 4.6. Let $j: \mathcal{H}_{1} \longrightarrow \mathcal{H}$ be an isometric inclusion. If $S, T \in \mathcal{B}^{+}(\mathcal{H})$, then

(1) $j\left(j^{*} S j\right)^{1 / 2} j^{*}=\left(j j^{*} S j j^{*}\right)^{1 / 2}$.

(2) $\left(j^{*} S j, j^{*} T j\right)_{\mathcal{B}^{+}\left(\mathcal{H}_{1}\right)}=\left(j j^{*} S j j^{*}, j j^{*} T j j^{*}\right)_{\mathcal{B}^{+}(\mathcal{H})}$.

Proof. For (1), check that both sides are positive square roots of $j j^{*} S j j^{*}$. For (2), let $\left(e_{m}\right)_{m}$ be an orthonormal basis for $\mathcal{H}_{1}$ and suppose that $\left(j e_{m}\right)_{m}$ and $\left(f_{n}\right)_{n}$ together make up an orthonormal basis for $\mathcal{H}$. Then

$$
\begin{aligned}
\left(j^{*} S j, j^{*} T j\right)_{\mathcal{B}^{+}\left(\mathcal{H}_{1}\right)} & =\sum_{m}\left\langle j^{*} T j\left(j^{*} S j\right)^{1 / 2} e_{m},\left(j^{*} S j\right)^{1 / 2} e_{m}\right\rangle_{\mathcal{H}_{1}} \\
& =\sum_{m}\left\langle j j^{*} T j j^{*} j\left(j^{*} S j\right)^{1 / 2} j^{*} j e_{m}, j\left(j^{*} S j\right)^{1 / 2} j^{*} j e_{m}\right\rangle_{\mathcal{H}} \\
& =\sum_{m}\left\langle j j^{*} T j j^{*}\left(j j^{*} S j j^{*}\right)^{1 / 2} j e_{m},\left(j j^{*} S j j^{*}\right)^{1 / 2} j e_{m}\right\rangle_{\mathcal{H}} \\
& =\left(j j^{*} S j j^{*}, j j^{*} T j j^{*}\right)_{\mathcal{B}^{+}(\mathcal{H})}
\end{aligned}
$$

since

$$
\left\langle j j^{*} T j j^{*}\left(j j^{*} S j j^{*}\right)^{1 / 2} f_{n},\left(j j^{*} S j j^{*}\right)^{1 / 2} f_{n}\right\rangle_{\mathcal{H}}=0
$$

for all $n$.

Lemma 4.7. Let $\mathcal{H}$ be a Hilbert space. If $X, Y \in \mathcal{B}(\mathcal{H})$ and $T \in \mathcal{B}^{+}(\mathcal{H})$, then

$$
(X+Y)^{*} T(X+Y) \leq 2\left(X^{*} T X+Y^{*} T Y\right)
$$


Proof.

$$
\begin{aligned}
\left\langle(X+Y)^{*} T(X+Y) v, v\right\rangle & =\left\|T^{1 / 2}(X v+Y v)\right\|^{2} \\
& \leq\left(\left\|T^{1 / 2} X v\right\|+\left\|T^{1 / 2} Y v\right\|\right)^{2} \\
& \leq 2\left(\left\|T^{1 / 2} X v\right\|^{2}+\left\|T^{1 / 2} Y v\right\|^{2}\right) \\
& =2\left(\left\langle X^{*} T X v, v\right\rangle+\left\langle Y^{*} T Y v, v\right\rangle\right) .
\end{aligned}
$$

For fixed $a \in A$ we must show that

$$
\left(\iota_{e}^{*} \tau_{+}^{\prime}\left(\mathbf{1}_{\Omega(a)}\right) \iota_{e}, \iota_{e}^{*} \tau_{+}^{\prime}\left(\mathbf{1}-\mathbf{1}_{\Omega(a)}\right) \iota_{e}\right)_{\mathcal{B}^{+}\left(\mathcal{W}_{e}\right)}
$$

is finite. Let $j_{e}: \mathcal{W}_{e} \longrightarrow \mathcal{H} \otimes \mathcal{V}$ be inclusion. Then

$$
\left(\iota_{+} \otimes \mathrm{id}\right) j_{e}=\iota_{e} \quad j_{e}^{*} j_{e}=\operatorname{id}_{\mathcal{W}_{e}} \quad 2 j_{e} j_{e}^{*}=\operatorname{id}_{\mathcal{H} \otimes \mathcal{V}}+(S \otimes s) .
$$

Together with these identities Lemma 4.6 says that up to a factor (4.4) equals

$$
\begin{aligned}
& \left((\mathrm{id}+(S \otimes s))^{*}\left(\iota_{+} \otimes \mathrm{id}\right)^{*} \tau_{+}^{\prime}\left(\mathbf{1}_{\Omega(a)}\right)\left(\iota_{+} \otimes \mathrm{id}\right)(\mathrm{id}+(S \otimes s)),\right. \\
& \left.(\mathrm{id}+(S \otimes s))^{*}\left(\iota_{+} \otimes \mathrm{id}\right)^{*} \tau_{+}^{\prime}\left(\mathbf{1}-\mathbf{1}_{\Omega(a)}\right)\left(\iota_{+} \otimes \mathrm{id}\right)(\mathrm{id}+(S \otimes s))\right)_{\mathcal{B}^{+}(\mathcal{H} \otimes \mathcal{V})} .
\end{aligned}
$$

By Lemma 4.7 the first of the two operators in this inner product is bounded by

$$
\begin{aligned}
& 2\left(\left(\iota_{+} \otimes \mathrm{id}\right)^{*} \tau_{+}^{\prime}\left(\mathbf{1}_{\Omega(a)}\right)\left(\iota_{+} \otimes \mathrm{id}\right)\right. \\
& \left.\quad+(S \otimes s)^{*}\left(\iota_{+} \otimes \mathrm{id}\right)^{*} \tau_{+}^{\prime}\left(\mathbf{1}_{\Omega(a)}\right)\left(\iota_{+} \otimes \mathrm{id}\right)(S \otimes s)\right) .
\end{aligned}
$$

Keeping in mind (5) of Lemma 4.3, the identity of $\tau_{+}^{\prime}$ and $\tau_{-}^{\prime}$ as representations of $C(\Omega)$ and the fact that $s^{*} s=\mathrm{id}$, one sees that (4.6) is equal to

$$
\begin{aligned}
2\left(\left(\iota_{+} \otimes \mathrm{id}\right)^{*} \tau_{+}^{\prime}\left(\mathbf{1}_{\Omega(a)}\right)\right. & \left.\left(\iota_{+} \otimes \mathrm{id}\right)+\left(\iota_{-} \otimes s\right)^{*} \tau_{-}^{\prime}\left(\mathbf{1}_{\Omega(a)}\right)\left(\iota_{-} \otimes s\right)\right) \\
& =2\left(\iota_{+}^{*} \pi_{+}^{\prime}\left(\mathbf{1}_{\Omega(a)}\right) \iota_{+} \otimes \mathrm{id}+\iota_{-}^{*} \pi_{-}^{\prime}\left(\mathbf{1}_{\Omega(a)}\right) \iota_{-} \otimes \mathrm{id}\right) .
\end{aligned}
$$

This bound, and the similar bound on the second operator in (4.5), together with the bilinearity and positivity of the $\mathcal{B}^{+}(\mathcal{H} \otimes \mathcal{V})$-inner product, show that up to a factor (4.5) is bounded by

$$
\begin{aligned}
&\left(\iota_{+}^{*} \pi_{+}^{\prime}\left(\mathbf{1}_{\Omega(a)}\right) \iota_{+} \otimes \mathrm{id}+\iota_{-}^{*} \pi_{-}^{\prime}\left(\mathbf{1}_{\Omega(a)}\right) \iota_{-} \otimes \mathrm{id},\right. \\
&\left.\iota_{+}^{*} \pi_{+}^{\prime}\left(\mathbf{1}-\mathbf{1}_{\Omega(a)}\right) \iota_{+} \otimes \mathrm{id}+\iota_{-}^{*} \pi_{-}^{\prime}\left(\mathbf{1}-\mathbf{1}_{\Omega(a)}\right) \iota_{-} \otimes \mathrm{id}\right)_{\mathcal{B}^{+}(\mathcal{H} \otimes \mathcal{V})} .
\end{aligned}
$$


This expands into four terms. The term

$$
\left(\iota_{+}^{*} \pi_{+}^{\prime}\left(\mathbf{1}_{\Omega(a)}\right) \iota_{+} \otimes \mathrm{id}, \iota_{+}^{*} \pi_{+}^{\prime}\left(\mathbf{1}-\mathbf{1}_{\Omega(a)}\right) \iota_{+} \otimes \mathrm{id}\right)_{\mathcal{B}^{+}(\mathcal{H} \otimes \mathcal{V})}
$$

is zero since the two operators involved are complementary orthogonal projections.

$$
\left(\iota_{+}^{*} \pi_{+}^{\prime}\left(\mathbf{1}_{\Omega(a)}\right) \iota_{+} \otimes \mathrm{id}, \iota_{-}^{*} \pi_{-}^{\prime}\left(\mathbf{1}-\mathbf{1}_{\Omega(a)}\right) \iota_{-} \otimes \mathrm{id}\right)_{\mathcal{B}^{+}(\mathcal{H} \otimes \mathcal{V})}
$$

is finite by Proposition 3.11. The other two terms are zero and finite respectively by symmetry. This concludes the proof that (4.4) is finite, and so concludes the proof of Theorem 4.4 for $\tau_{e}$. To prove the (FTC) for $\left(\iota_{o}, \tau_{+}^{\prime}\right)$ with itself use the same method, letting $j_{o}: \mathcal{W}_{o} \longrightarrow \mathcal{H} \otimes \mathcal{V}$ be inclusion and making use of

$$
\left(\iota_{+} \otimes \mathrm{id}\right) j_{o}=\iota_{o} \quad j_{o}^{*} j_{o}=\mathrm{id}_{\mathcal{W}_{o}} \quad 2 j_{o} j_{o}^{*}=\operatorname{id}_{\mathcal{H} \otimes \mathcal{V}}-(S \otimes s) .
$$

\section{References}

J. Dixmier, Von Neumann Algebras, North-Holland, Amsterdam, 1981.

A. Figà-Talamanca and C. Nebbia, Harmonic Analysis and Representation Theory for Groups Acting on Homogeneous Trees, London Mathematical Society Lecture Note Series, 162, Cambridge University Press, Cambridge, 1991.

A. Figà-Talamanca and T. Steger, Harmonic analysis for anisotropic random walks on homogeneous trees, to appear in Mem. Amer. Math. Soc.

G. Kuhn and T. Steger, More irreducible representations of free groups, to appear in Duke Math. J.

M. Reed and B. Simon, Methods of Modern Mathematical Physics I: Functional Analysis, Academic Press, New York 1972.

T. Steger, Free group representations: duplicity on the boundary, in preparation.

Receiver June 6, 1993 and revised April 7, 1994.

Istituto di Matematica E Fisica

Via Vienna 2, 07100 SASSARI, ItAlia

E-mail address: pensa@ssmain.uniss.it steger@ssmain.uniss.it 


\title{
PACIFIC JOURNAL OF MATHEMATICS
}

Founded by

\author{
$\begin{array}{ll}\text { E. F. Beckenbach (1906-1982) F. Wolf (1904-1989) } & \text { F }\end{array}$
}

EDITORS

Sun-Yung A. Chang (Managing Editor) Robert Finn

University of California

Los Angeles, CA 90095-1555

pacific@math.ucla.edu

F. Michael Christ

University of California

Los Angeles, CA 90095-1555

christ@math.ucla.edu

Nicholas Ercolani

University of Arizona

Tucson, AZ 85721

ercolani@math.arizona.edu
Stanford University

Stanford, CA 94305

finn@gauss.stanford.edu

Steven Kerckhoff

Stanford University

Stanford, CA 94305

spk@gauss.stanford.edu

Martin Scharlemann

University of California

Santa Barbara, CA 93106

mgscharl@math.ucsb.edu
Gang Tian

Massachusettes Institute of Technology

Cambridge, MA 02139

tian@math.mit.edu

\section{S. Varadarajan}

University of California

Los Angeles, CA 90095-1555

vsv@math.ucla.edu

Dan Voiculescu

University of California

Berkeley, CA 94720

dvv@math.berkeley.edu

\section{SUPPORTING INSTITUTIONS}

\author{
CALIFORNIA INSTITUTE OF TECHNOLOGY \\ NEW MEXICO STATE UNIVERSITY \\ OREGON STATE UNIVERSITY \\ STANFORD UNIVERSITY \\ UNIVERSITY OF ARIZONA \\ UNIVERSITY OF BRITISH COLUMBIA \\ UNIVERSITY OF CALIFORNIA \\ UNIVERSITY OF HAWAII
}

\author{
UNIVERSITY OF MONTANA \\ UNIVERSITY OF NEVADA, RENO \\ UNIVERSITY OF OREGON \\ UNIVERSITY OF SOUTHERN CALIFORNIA \\ UNIVERSITY OF UTAH \\ UNIVERSITY OF WASHINGTON \\ WASHINGTON STATE UNIVERSITY
}

The supporting Institutions listed above contribute to the cost of publication of this Journal, but they are not owners or publishers and have no responsibility for its contents or policies.

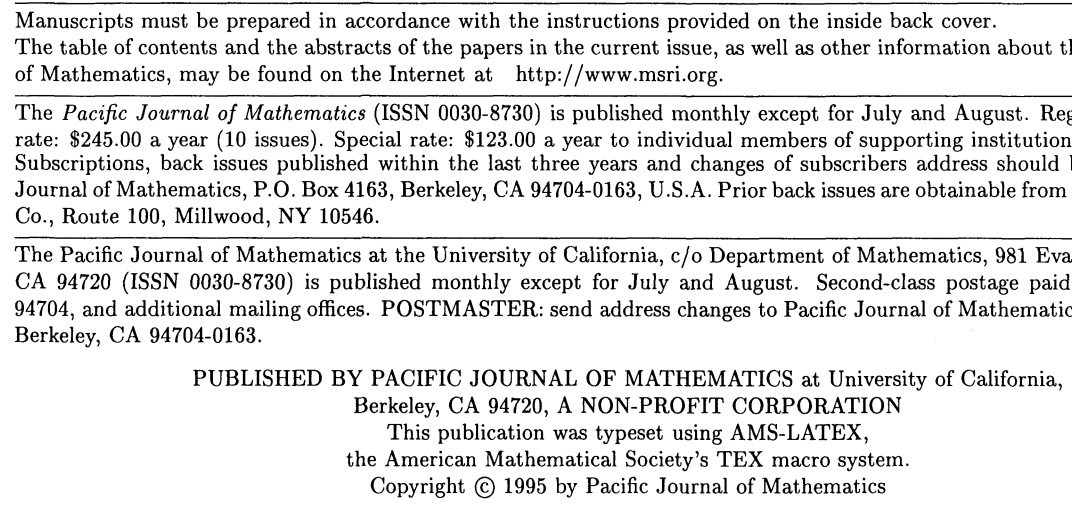




\section{PACIFIC JOURNAL OF MATHEMATICS}

\section{Volume $173 \quad$ No. $1 \quad$ March 1996}

Isometric immersions of $H_{1}^{n}$ into $H_{1}^{n+1}$

KINETSU ABE

Rotationally symmetric hypersurfaces with prescribed mean curvature

MARIE-FRANÇOISE BIDAUT-VÉRON

The covers of a Noetherian module

JIAN-JUN CHUAI

On the odd primary cohomology of higher projective planes

MARK FoSKEY and MiCHAEL DAVID SLACK

Unit indices of some imaginary composite quadratic fields. II

MiKinito HiRABAYASHI

Mixed automorphic vector bundles on Shimura varieties

MIN Ho LeE

Trace ideal criteria for Toeplitz and Hankel operators on the weighted Bergman

spaces with exponential type weights

PENG LIN and RICHARD ROCHBERG

On quadratic reciprocity over function fields

KATHY DONOVAN MERRILL and LYNNE WALLING

$\left(A_{2}\right)$-conditions and Carleson inequalities in Bergman spaces

TAKAHIKO NAKAZI and MASAHIRO YAMADA

A note on a paper of E. Boasso and A. Larotonda: "A spectral theory for solvable Lie 173 algebras of operators"
C. Отт

Tensor products with anisotropic principal series representations of free groups

Carlo Pensavalle and Tim Steger

On Ricci deformation of a Riemannian metric on manifold with boundary

YING SHEN

The Weyl quantization of Poisson $S U(2)$

Albert Jeu-Liang Sheu

Weyl's law for $S L(3, \mathbb{Z}) \backslash S L(3, \mathbb{R}) / S O(3, \mathbb{R})$

ERIC GeORge Stade and Dorothy IRENe Wallace (ANDREOli)

Minimal hyperspheres in two-point homogeneous spaces

PER TOMTER

Subalgebras of little Lipschitz algebras

NiKOLAI ISAAC WEAVER 